\title{
Recombinant Growth Factor Gene Expression in Vascular Cells in Vivo
}

\author{
ELIZABETH G. NABEL,,${ }^{a}$ GREGORY E. PLAUTZ, ${ }^{b}$ AND \\ GARY J. NABEL ${ }^{a, c, d}$
}

\author{
Departments of ${ }^{a}$ Internal Medicine, 'Biological Chemistry, \\ and ${ }^{b}$ Pediatrics \\ ${ }^{\text {'Howard Hughes Medical Institute }}$ \\ University of Michigan Medical Center \\ Ann Arbor, Michigan 48109-0688
}

Gene transfer is the introduction of foreign DNA into host cells or tissues. Gene transfer can be performed to evaluate the pathophysiology of a disease process, for example, the function of gene in a cell or organ, and to provide a gene therapy for a missing or defective native gene. Transgenic animals gain new genetic information from the addition of foreign DNA to cells that are maintained in the germ line and subsequently expressed in somatic tissues. ${ }^{1}$ Gene transfer differs in that recombinant DNA is introduced into somatic cells of animals without transmission into the germ line. ${ }^{2}$ Somatic cell gene transfer can be employed to examine the expression and regulation of a gene in a particular tissue or organ and to deliver a foreign gene to an animal to replace or complement a missing or defective gene.

For several years we have been interested in developing gene transfer methods to directly introduce new genes into vascular cells in vivo in order to study the pathophysiology of vascular disease and to develop gene therapies for cardiovascular diseases. Two strategies are employed to introduce foreign genes into vascular cells: cell-mediated and direct gene transfer. Cell transfer is a multistep process in which autologous target cells are removed from the animal, gene transduction and selection are performed in culture, and genetically modified cells are reimplanted into the animal. Cell-mediated gene transfer permits the analysis of a gene product in a specific tissue. However, cell-mediated gene transfer is technically cumbersome, costly, and complex. Direct gene transfer is the transmission of foreign DNA directly into cells or organs in vivo, eliminating prior cell transduction in tissue culture. Direct gene transfer simplifies the procedure considerably; however, at the present time, direct gene transfer into vascular cells lacks cell-specific expression vectors and regulated gene expression (TABLE 1).

\section{GENE TRANSFER VECTORS}

Several viral and nonviral vectors have been used successfully to introduce foreign DNA into vascular cells in vitro and in vivo. The advantages and limitations of these vectors, which include retrovirus, adenovirus, adenoviral conjugates, and cationic liposomes, have been reviewed elsewhere. ${ }^{3}$ In general, transduction of vascular cells in vivo with replication-incompetent retroviral vectors results in 
TABLE 1. Requirements for Gene Therapy

- Efficient transduction system

- Stable, high-level expression of gene product

- Tissue-specific expression system

- Minimal risk of mutagenesis and persistent infection of the recipient

transfection efficiencies of $0.1-1 \% .^{4,5}$ Retroviral vectors transduce primarily dividing cells and integrate into host chromosome in a random manner. These vectors may not be suitable to express genes in postmitotic cells, such as cardiac myocytes. Previous studies have shown that retroviral infection can be inhibited in replicating cells and culture, and that such infected cells must be replicating at the time of infection for successful gene transfer. ${ }^{6}$ A potential complication of retroviral infection is recombination to a wild-type virus which could replicate in an uncontrolled manner in the host organism. To minimize this potential complication, viral gene products required for replication are deleted from the retroviral sequence and introduced into packaging cell lines. ${ }^{7.8}$ When the retrovirus is cotransfected in packaging cell lines, a viral particle capable of infection is produced; however, the virion cannot replicate. This reduces the possibility that the retrovirus will duplicate after introduction into the host cell. ${ }^{9}$ Retroviral gene transfer has been performed directly in pig arteries without detection of helper-virus activity up to five months after transduction, ${ }^{4}$ suggesting that this vector is relatively safe.

Transfection employing cationic liposomes has been commonly used to achieve high efficiency in cultured cells. Development of commercialized forms of cationic liposomes in the late 1980 s resulted in their broad use for in vitro and in vivo transfection. ${ }^{10}$ Cationic lipid vesicles condense with DNA to form complexes in which the DNA is trapped. " Because virtually all biological surfaces have a net negative charge, the positively charged lipid vesicles containing polynucleotide interact spontaneously with these surfaces, delivering polynucleotide to the cell. Cationic lipid/polynucleotides fuse with cell membranes in such a manner that the entrapped DNA avoids degradation in the liposomal component. ${ }^{12}$ After delivery to the cytoplasm, plasmid DNA translocates to the nucleus and persists in the nucleus predominantly as an episome. Integration into host chromosome occurs at a low frequency. Transient expression levels and stable transformation efficiencies depend upon the expression vector and cell lines. ${ }^{13}$ Several technical factors are also important in cationic liposome transfection including the ratio of liposome to DNA, the cationic liposome formulation, and the use of serumfree conditions. The advantages of cationic liposome transfection include safety, reproducibility, and ease of preparation. Safety concerns about the use of retrovirus or adenovirus in humans are largely mitigated by this nonviral vector.

Recombinant adenoviruses have several properties that make them attractive vectors for in vivo gene delivery, including high-efficiency gene transfer. Several studies have reported in vivo gene delivery using adenovirus into vascular cells with efficiencies of $>50 \%$ of vascular cells. ${ }^{14}$ This high efficiency results from effective propagation of adenoviral stocks to high titer, the promiscuity and tissue trophism of adenoviral infection of cells, and the infectivity of adenovirus for replicating and nonreplicating cells. ${ }^{15}$ In dividing cells, adenoviral sequences integrated low frequency into host chromosomes. However, in nondividing somatic cells in vivo, the adenoviral genome appears to be maintained as an episome. ${ }^{16}$ Several issues relevant to the application of adenoviral vectors to human therapies 
are currently being investigated, including stability of gene expression, host-cell immune responses, transmission to germ-line cells, and toxicity.

\section{GENE TRANSFER INTO VASCULAR CELLS}

For several years, we have been interested in developing gene transfer to directly introduce new genes into vascular cells in vivo in order to study the pathophysiology of vascular disease. In particular, we have been interested in investigating the mechanisms of cellular proliferation in arteries after expression of growth factor and cytokine genes in arteries in vivo. A variety of growth factors have been reported to stimulate vascular cell proliferation and vessel formation in vivo. ${ }^{17-20}$ Although the genes encoding many factors have been cloned and their mechanism of action defined in vitro, definition of their role in vivo has been more difficult to analyze. To address this question we introduced recombinant genes for three growth factors, PDGF B, secreted FGF-1 (acidic FGF), and active TGF- $\beta 1$, into pig arteries in vivo using gene transfer (TABLE 2). The advantage of this system is that a recombinant growth factor gene can be transfected into a local artery segment where its expression and effects on arterial function can be evaluated. ${ }^{4.21}$

PDGF BB is a potent smooth muscle cell mitogen and smooth muscle chemoattractant. ${ }^{22,23}$ The function of the PDGF $B$ gene in tissue injury and restenosis in vivo has been more difficult to address. To investigate the in vivo function of a PDGF $B$ gene, we transfected a plasmid expression vector encoding a human PDGF $B$ gene into porcine arteries. ${ }^{24}$ Transfer of plasma DNA was documented by PCR; an expression of mRNA was confirmed by reverse transcriptase PCR. Recombinant PDGF BB protein was detected in transduced arteries by immunohistochemistry using a monoclonal antibody against human PDGF BB. Porcine arteries transduced with PDGF BB demonstrated severe intimal thickening, characterized by increased cellularity and smooth muscle cell proliferation in contrast to control arteries transduced with a reporter gene. Quantitative morphometry confirmed a significant difference in intimal-to-medial ratio between PDGF B and control arteries. Further studies are investigating the mechanisms of PDGF B-induced intimal hyperplasia, including proliferation kinetics.

The heparin binding fibroblast growth factor family has proliferative and angiogenic properties in vivo. ${ }^{25.26}$ To investigate the role of FGF-1 in vascular pathology, we transfected a plasmid expression vector encoding for secreted FGF- 1 into

TABLE 2. Gene Expression in the Arterial Wall

\begin{tabular}{lllll}
\hline \multicolumn{1}{c}{ Gene } & DNA & mRNA & Protein & \multicolumn{1}{c}{ Biological Effect } \\
\hline $\begin{array}{l}\text { HLA-B7 } \\
\text { Retrovirus }\end{array}$ & +++ & n.d. & +++ & Vasculitis \\
Plasmid & +++ & n.d. & +++ & Vasculitis \\
$\begin{array}{l}\text { PDGF-B } \\
\text { Plasmid }\end{array}$ & +++ & +++ & +++ & $\begin{array}{l}\text { Cellular proliferation } \\
\text { Intimal hyperplasia }\end{array}$ \\
$\begin{array}{l}\text { FGF-1 (secreted) } \\
\text { Plasmid }\end{array}$ & +++ & +++ & +++ & $\begin{array}{l}\text { Angiogenesis } \\
\text { Intimal thickening }\end{array}$ \\
$\begin{array}{l}\text { TGF- } \beta \text { 1 (active) } \\
\text { Plasmid }\end{array}$ & +++ & +++ & +++ & $\begin{array}{l}\text { Extracellular matrix synthesis } \\
\text { Intimal thickening }\end{array}$ \\
\hline
\end{tabular}


TABLE 3. Future Directions

- Improved efficiency of gene transfection

- Cell-specific expression of vectors

- Regulated gene expression

- Definition of therapeutic genes

- Development of catheters for genetic technologies

porcine arteries. ${ }^{27}$ Transfer of the plasmid and expression of recombinant mRNA and protein were determined by appropriate PCR and immunohistochemistry studies, respectively. Intimal thickening was also observed in FGF-1-transduced arteries. However, in contrast to vessels transduced with other growth factor genes, FGF-1 vessels with expanded intima demonstrated neocapillaries within the intima. These findings provide a role for FGF-1 in stimulating vascular angiogenesis in vivo.

In more recent studies, the role of TGF- $\beta 1$ in normal arteries has been explored after transfection of a plasmid expression vector encoding an active form of TGF$\beta 1$ into normal porcine arteries. In these studies, ${ }^{28}$ we found that TGF- $\beta 1$ is associated with increased procollagen and collagen synthesis in the media and intima of TGF- $\beta 1-$ transfected arteries, in contrast to control arteries transfected with a reporter gene.

Findings from these experiments which have examined the in vivo function of three growth factor genes suggest that expression of a recombinant growth factor gene can have direct and indirect effects on vascular function in vivo. For example, expression of PDGF BB is associated with intense intimal cellular proliferation, and FGF-1 may play a role in stimulating vascular angiogenesis, whereas TGF$\beta 1$ is associated with increased extracellular matrix production. Despite these different patterns of gene expression, all three growth factor genes produced some intimal thickening. These findings suggest that intimal thickening may represent a common response to gene expression of multiple growth factors that each in turn exert distinct effects on vessel repair. On the basis of these studies examining the function of growth factor genes in vivo, animal models of vascular disease can be developed and molecular targets can be tested.

\section{SUMMARY}

Several issues are important to the use of direct gene transfer as an investigative tool and as a potential therapeutic modality (TABLE 3). Transfection efficiencies of different vectors must be improved and optimized. Retroviral vectors and DNA liposome conjugates currently used in animal models are low-efficiency vectors. Adenoviruses and adenoviral conjugates appear promising, but issues related to gene persistence, germ-line transmission, and stability of expression must be explored. Second, the pharmacology or dose-response properties of recombinant gene expression have not been investigated. It is not currently known how many cells must be transfected in an arterial segment in order to produce a desired biological effect. Our studies suggest that only a small population of cells is required to secrete a recombinant gene product into the local milieu. This gene product may then have local paracrine effects with amplification of the biological response, suggesting a "gain of function." Third, methods must be developed to target recombinant genes specifically to endothelial cells or smooth muscle cells using cell-specific promoters. Finally, gene expression should be regulated through inducible or repressible promoters. 
Nonetheless, during the past ten years a dramatic expansion in the fields of gene transfer and gene therapy has occurred. We have entered a new era in which molecular genetic techniques are being increasingly used to investigate the pathophysiology of cardiovascular disorders and to design potential therapies for these diseases. Although technical hurdles related to optimization of vectors and regulated gene expression must be solved, molecular genetic approaches will be increasingly used to study and treat cardiovascular diseases.

\section{REFERENCES}

1. Palmiter, R. D. \& R. L. Brinster. 1985. Transgenic mice. Cell 41: 343-354.

2. Friedman, T. 1989. Progress toward human gene therapy. Science 244: 1275-1281.

3. Nabel, E. G. \& G. J. Nabel. 1994. Complex models-novel interaction for the study of cardiovascular biology. Annu. Rev. Physiol. 56: 741-761.

4. Nabel, E. G., G. Plautz \& G. J. Nabel. 1990. Site-specific gene expression in vivo by direct gene transfer into the arterial wall. Science 249: 1285-1288.

5. Leclerc, G., D. Gal, S. TAkeshita, S. Nikol, L. Weir \& J. M. Isner. 1992. Percutaneous arterial gene transfer in a rabbit model. Efficiency in normal and balloondilated atherosclerotic arteries. J. Clin. Invest. 90: 936-944.

6. Miller, D. G., M. A. Adam \& A. D. Miller. 1990. Gene transfer by retrovirus vectors occurs only in cells that are actively replicating at the time of infection. Mol. Cell. Biol. 10: 4239-4242.

7. Cone, R. D. \& R. C. Mulligan. 1984. High-efficiency gene transfer into mammalian cells: Generation of helper-free recombinant retrovirus with broad mammalian host range. Proc. Natl. Acad. Sci. USA 81: 6349-6353.

8. Danos, O. \& R. C. Mulligan. 1988. Safe and efficient generation of recombinant retroviruses with amphotropic and ecotropic host ranges. Proc. Natl. Acad. Sci. USA 85: 6460-6464.

9. Miller, A. D. \& C. Buttimore. 1986. Redesign of retrovirus packaging cell lines to avoid recombination leading to helper virus production. Mol. Cell. Biol. 6: 2895-2902.

10. Felgner, P. L. \& G. M. Ringold. 1989. Cationic liposome-mediated transfection. Nature 337: 387-388.

11. Malone, R. W., P. L. Felgner \& I. M. Verma. 1989. Lipofectin mediated RNA transfection. Proc. Natl. Acad. Sci. USA 86: 6077-6081.

12. Duzgunes, N., J. A. Goldstein, D. S. Friend \& P. L. Felgner. 1989. Fusion of liposomes containing a novel cationic lipid, N-[2,3-(dioleyloxy)propyl]-N,N,N-trimethylammonium: Induction by multivalent anions and asymmetric fusion with acidic phospholipid vesicles. Biochemistry 28: 9179-9184.

13. Felgner, P. L. \& G. Rhodes. 1991. Gene therapeutics. Nature 349: 351-352.

14. Lemarchand, P., M. Jones, I. Yamada \& R. G. CRYSTal. 1993. In vivo gene transfer and expression in normal uninjured blood vessels using replication-deficient recombinant adenovirus vectors. Circ. Res. 72: 1132-1138.

15. Philipson, L., K. Lonberg-Holm \& U. Pettersson. 1968. Virus-receptor interaction in an adenovirus system. J. Virol. 2: 1064-1075.

16. Horwitz, M. S. 1990. Virology. Raven Press. New York, NY.

17. Ferns, G. A. A., E. W. Raines, K. H. Sprugel, A. S. Motani, M. A. Reidy \& R. Ross. 1991. Inhibition of neointimal smooth muscle accumulation after angioplasty by an antibody to PDGF. Science 253: 1129-1132.

18. Jawien, A., D. F. Bowen-Pope, V. Lindner, S. M. Schwartz \& A. W. Clowes. 1992. Platelet-derived growth factor promotes smooth muscle migration and intimal thickening in a rat model of balloon angioplasty. J. Clin. Invest. 89: 507-511.

19. Burgess, W. H. \& T. Maciag. 1989. The heparin-binding (fibroblast) growth factor family of proteins. Annu. Rev. Biochem. 58: 575-606.

20. Roberts, A. N., M. B. Sporn, R. K. Assolan, J. M. Smith, N. S. Roche, L. M. 
Wakefield, U. I. Heine, L. A. Liotta, V. Falanga, J. H. Kehrl \& A. S. Fauci. 1986. Transforming growth factor type $\beta$ : Rapid induction of fibrosis and angiogenesis in vivo and stimulation of collagen formation in vitro. Proc. Natl. Acad. Sci. USA 83: 4167-4171.

21. Nabel, E. G., G. Plautz \& G. J. Nabel. 1992. Transduction of a foreign histocompatibility gene into the arterial wall induces vasculitis. Proc. Natl. Acad. Sci. USA 89: 5157-5161.

22. Ross, R., J. Glomset, B. Kariya \& L. Harker. 1974. A platelet-dependent serum factor that stimulates the proliferation of arterial smooth muscle cells in vitro. Proc. Natl. Acad. Sci. USA 71: 1207-1210.

23. Grotendorst, G., H. E. J. Seppa, H. K. Kleinman \& G. R. Martin. 1982. Attachment of smooth muscle cells to collagen and their migration toward platelet-derived growth factor. Proc. Natl. Acad. Sci. USA 71: 3669-3672.

24. Nabel, E. G., Z. Yang, S. Liptay, H. San, D. Gordon, C. C. Haudenschild \& G. J. NABEL. 1993. Recombinant platelet-derived growth factor B gene expression in porcine arteries induces intimal hyperplasia in vivo. J. Clin. Invest. 91: 1822-1829.

25. Agraham, J. A., A. Mergia, J. L. Whang, A. Tumolo, J. Friedman, K. A. Huerrild, D. Gospodarowicz \& J. C. FidDEs. 1986. Nucleotide sequence of a bovine clone encoding the angiogenic protein, basic fibroblast growth factor. Science 233: $545-548$.

26. Ausprunk, D. H. \& J. Folkman. 1977. Migration and proliferation of endothelial cells in preformed and newly formed blood vessels during tumor angiogenesis. Microvasc. Res. 14: 53-65.

27. Nabel, E. G., Z. Yang, G. Plautz, R. Forough, X. Zhan, C. C. Haudenschild, T. MaCiag \& G. J. Nabel. 1993. Recombinant fibroblast growth factor-1 promotes intimal hyperplasia and angiogenesis in arteries in vivo. Nature 362: 844-846.

28. Nabel, E. G., L. Shum, V. J. Pompili, Z. Y. Yang, H. San, H. B. Shu, S. Liptay, D. Gordon, R. DERyNCK \& G. J. NABEL. 1993. Direct gene transfer of transforming growth factor $\beta 1$ into arteries stimulates fibrocellular hyperplasia. Proc. Natl. Acad. Sci. USA. 90: 10759-10763. 